\title{
Restauración estética de dientes temporales anteriores con coronas de zirconia. Caso clínico (seguimiento a 24 meses)
}

\author{
Aesthetic restoration of anterior temporary teeth with zirconia crowns. \\ Case report (24-month follow-up)
}

\author{
Brianda González-Escalante, ${ }^{1}$ Carla Mariana Muciño-Malváez ${ }^{1}$ y Laura Elena Allende-Trejo ${ }^{2}$
}

\footnotetext{
${ }^{1}$ Alumna de la Especialización en Estomatología del Niño y del Adolescente, FES Zaragoza UNAM.

${ }^{2}$ Profesora de la Especialización en Estomatología del Niño y del Adolescente, FES Zaragoza, UNAM.
}

\section{RESUMEN}

Introducción. El reto de brindar tratamientos estéticos a edades tempranas y poder dar un seguimiento a su evolución es cada vez más frecuente, sobre todo cuando se trata de los dientes temporales anteriores. Las coronas de zirconia pueden ser una alternativa, sin embargo, es poco conocida su eficacia a largo plazo en pacientes pediátricos. Por tal motivo, el propósito del estudio es mostrar la evolución a 24 meses de la restauración con coronas de zirconia en dientes anteriores deciduos, incluyendo el diagnóstico, rehabilitación y seguimiento semestral hasta el recambio de dentición. Caso clínico. Paciente masculino de 4 años 3 meses; acude a consulta en 2016 por presentar múltiples lesiones cariosas, restaurando los dientes temporales anteriores del maxilar superior con coronas estéticas de zirconia. Después de realizar una evaluación clínica y radiográfica se programó la exodoncia de los dientes 61 y 62 , por destrucción coronaria y presencia de absceso crónico con reabsorción interna, lo cual limitó la posibilidad de conservarlos y fue necesario la colocación de un mantenedor de espacio estético; sin embargo los dientes 51 y 52, presentaron lesión de caries inactiva de $2^{\circ}$ grado con tejido pulpar y periapical sano, los cuales fueron rehabilitados con coronas de zirconia NuSmile $\mathrm{Zr}$, verificando radiográficamente su ajuste y sellado. La primera evaluación postoperatoria de las coronas de zirconia se realizó a la semana de su colocación, después el seguimiento clínico y radiográfico fue a los seis, doce, dieciocho y veinticuatro meses, logrando tener un acompañamiento de la restauración hasta el momento del recambio de dentición Conclusiones. Las coronas de zirconia son una buena opción de tratamiento que no requiere en todos los casos de una terapia pulpar previa, no afecta el proceso de exfoliación y proporciona estabilidad emocional y seguridad en los pacientes infantiles que presentan afectaciones severas de los dientes anteriores como consecuencia de la enfermedad caries.

Palabras clave: Caries de la primera infancia, restauración estética, coronas de zirconia.

\begin{abstract}
Introduction. The challenge of providing aesthetic treatments at an early age and being able to monitor their evolution is becoming more and more frequent, especially when it comes to anterior primary teeth. Zirconia crowns can be an alternative, however, their long-term efficacy in pediatric patients is poorly understood. Hence, the purpose of the study is to show the 24-month evolution of the restoration with zirconia crowns in deciduous anterior teeth, including the diagnosis, rehabilitation and biannual follow-up up to the replacement of the dentition. Clinical case. A 4 year, 3 month old male patient who attended the consultation in 2016 due to multiple carious lesions, restoring the upper jaw anterior temporary teeth with aesthetic zirconia crowns. After carrying out a clinical and radiographic evaluation, the extraction of teeth 61 and 62 was programmed due to coronary destruction and the presence of chronic abscess with internal resorption which limited the possibility of conserving them and it was necessary to place an aesthetic space maintainer; However, teeth 51 and 52 presented a 2nd degree inactive caries lesion with healthy pulp and periapical tissue, which were rehabilitated with NuSmile Zr zirconia crowns, radiographically verifying their fit and sealing. The first postoperative evaluation of the zirconia crowns was performed a week after their placement. Then, the clinical and radiographic follow-up was at six, twelve, eighteen and twenty-four months, achieving a follow-up of the restoration up to the time for the dentition replacement. Conclusions. Zirconia crowns are a good treatment option that does not require prior pulp therapy in all cases. It does not affect the exfoliation process and provides emotional stability and safety in child patients with severe anterior tooth damage as a result of caries disease.
\end{abstract}

Key words: Early childhood caries, aesthetic restorations, zirconia crowns.
Correspondencia: Laura Elena Allende-Trejo

Email: coor.nino.adoles@zaragoza.unam.mx

Artículo recibido: 6 de septiembre de 2021

Artículo aceptado: 3 de noviembre de 2021
González-Escalante B, Muciño-Malváez CM y Allende-Trejo LE.Restauración estética de dientes temporales anteriores con coronas de zirconia. Caso clínico (seguimiento a 24 meses) CyRS. 2021; 3(2):20-25

DOI: https://doi.org/10.22201/fesz.26831422e.2021.3.2.3 


\section{INTRODUCCIÓN}

En los últimos años la forma en la que se conceptualiza a las restauraciones para los dientes deciduos se ha modificado, encontrando cada vez una mayor tendencia hacia la búsqueda de alternativas que cubran las expectativas estéticas de los niños, así como de sus padres. ${ }^{1}$

Lo anterior puede estar sujeto a los cambios en el comportamiento social que los niños viven a diario en su entorno familiar y escolar; pues en la actualidad es más frecuente encontrar a niños en edad temprana que presentan enfermedades bucales como la caries, la cual sigue siendo un problema de salud pública en todo el mundo y ha demostrado tener un impacto significativo en el bienestar emocional y la calidad de vida de los niños, lo cual puede tener desenlaces complicados como la pigmentación o ruptura del esmalte, el compromiso de infección y en el peor de los escenarios la pérdida prematura del órgano dentario. ${ }^{2-4}$

Para el estomatólogo pediatra representa un reto cada vez más frecuente la rehabilitación, ya que son los padres quienes demandan estándares altos por cumplir en la restauración dental de sus hijos. ${ }^{4,5}$

La estética, la toxicidad, la durabilidad y el costo son factores comunes que los padres consideran antes de dar su consentimiento para cualquier técnica de restauración. ${ }^{6,7}$

Los primeros dientes en ser afectados por caries de la primera infancia son los que se encuentran en el sector superior anterior; la pérdida temprana de uno o varios de éstos dientes temporales genera diversas complicaciones que redundan no sólo en la erupción de la dentición permanente, sino que afecta funciones como la fonación, la deglución, la masticación, así como el desarrollo de hábitos no funcionales y maloclusiones; por tal motivo es importante recuperar cada una de ellas sin olvidar la apariencia estética. ${ }^{8-11}$

En la última década ha aumentado el uso de coronas preformadas de zirconia para la restauración de dientes temporales anteriores, las cuales cumplen con todas las expectativas estéticas y funcionales que se requiere para una correcta restauración. Sin embargo, se puede presentar un desgaste fisiológico de los órganos dentarios temporales, debido a la dureza de la zirconia. Otra complicación que se ha reportado es el desgaste excesivo como consecuencia al proceso de adaptación, siendo necesario realizar en algunos casos tratamiento pulpar previo, con el objetivo de prevenir patologías pulpares irreversibles que conlleven a un fracaso del tratamiento restaurativo. ${ }^{12-15}$

Por lo anterior, el propósito del presente estudio es mostrar la evolución clínica a 24 meses de la restauración con coronas de zirconia en dientes anteriores deciduos, incluyendo el diagnóstico, rehabilitación y seguimiento hasta el recambio de dentición.

\section{Caso clínico}

\section{AnteCedentes}

Paciente masculino de cuatro años tres meses de edad aparentemente sano, se mostró cooperativo (tipo III en la escala de comportamiento de Frankl), que acude a consulta dental, por presentar múltiples lesiones cariosas. Al interrogatorio indirecto no refiere antecedentes personales patológicos y cuenta con esquema de vacunación completo. Refiere vivir en casa propia con todos los servicios, no tiene contacto con mascotas, toma baño diario con cambio de ropa, su higiene bucal es deficiente en frecuencia y técnica, lleva una dieta desequilibrada y de consistencia blanda con un alto contenido de carbohidratos.

Durante la exploración se identifica un biotipo mesocéfalo, perfil convexo, simetría facial, cuello cilíndrico con movimientos de hiperextensión y lateralidad adecuados, sin hallazgos de adenopatías, ATM sin alteración a los movimientos de apertura y cierre, mucosa peribucal y labios hidratados, clasificación Mallanpati II, hipertrofia amigdalina I, úvula paladar blando y paladar duro bien definido e insertado, mucosa yugal hidratada color rosa brillante, lengua con papilas definidas, piso de boca hidratado y vascularizado, glándulas salivales permeables y frenillos con buena inserción, forma de arcos oval, tipo I de Baume con dentición temporal completa y plano terminal mesial bilateral.

\section{Diagnóstico}

El diagnóstico estomatológico fue caries múltiple de diversos grados y patología pulpar en el cincuenta por ciento de su dentición; así como alteraciones funcionales tales como deglución atípica, respiración bucal, 


\section{( $@$ RevistaCyRS}

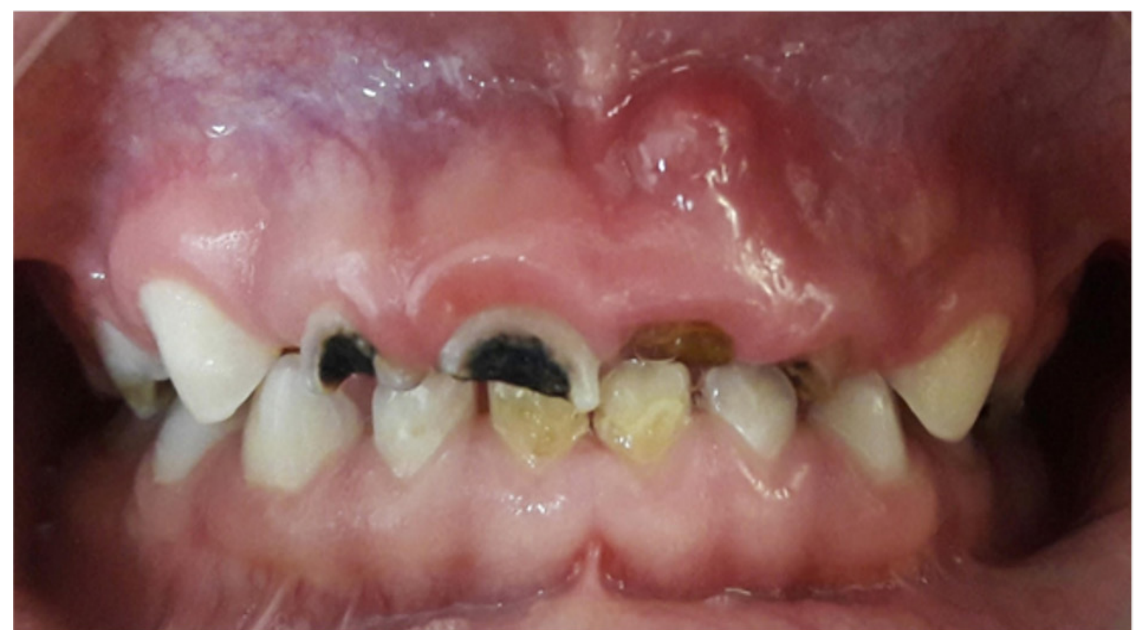

Figura 1. Fotografía inicial vista frontal de arcada superior e inferior con presencia de múltiples lesiones cariosas de diversos grados y presencia de absceso periapical crónico en diente 61 y 62.

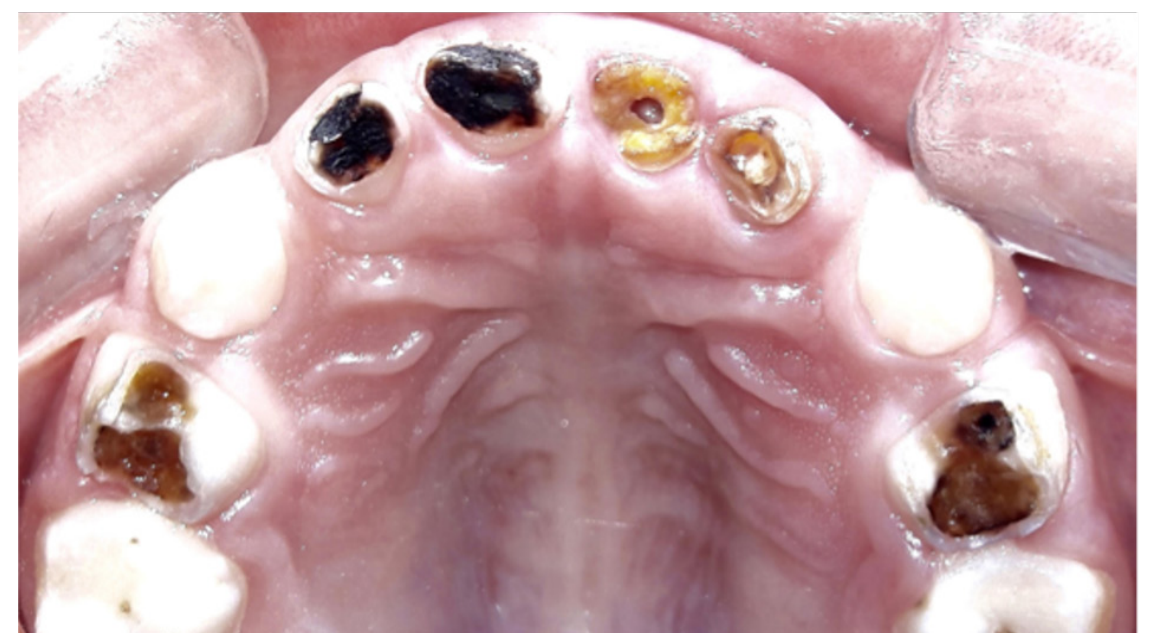

Figura 2. Fotografía inicial con vista oclusal de arcada superior e inferior con presencia de lesiones cariosas de múltiples grados.

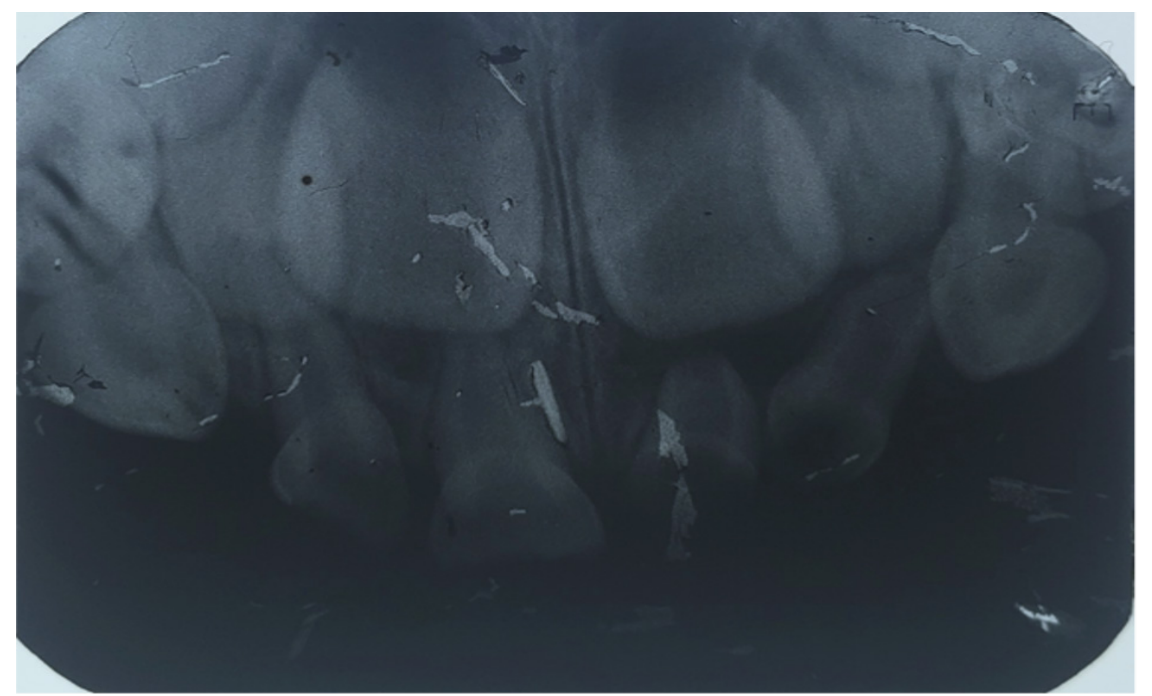

Figura 3. Fotografía de imagen radiográfica toma oclusal de zona anterosuperior, destacando los dientes 61 y 62 con presencia de reabsorción interna y destrucción coronaria. dolor a la masticación, afectación en el lenguaje y la estética (Figuras 1 y 2).

\section{TRATAMIENTO}

El tratamiento fue establecido en tres fases, se inició con la restauración del sector postero superior, seguida de los cuadrantes inferiores y se finalizó con el abordaje del sector anterosuperior.

Después de realizar una evaluación clínica y radiográfica se programó la exodoncia de los dientes 61 y 62 (Figura 3 ), por destrucción coronaria y presencia de absceso crónico con reabsorción interna, lo cual limitó la posibilidad de conservarlos y fue necesario la colocación de un mantenedor de espacio estético; sin embargo los dientes 51 y 52 , presentaron lesión de caries inactiva de $2^{\circ}$ grado con tejido pulpar y periapical sano, los cuales fueron rehabilitados con coronas de zirconia NuSmile $\mathrm{Zr}$, verificando radiográficamente su ajuste y sellado (Figura 4).

La primera evaluación postoperatoria de las coronas de zirconia se realizó a la semana de su colocación, después el seguimiento clínico y radiográfico fue a los seis, doce, dieciocho y veinticuatro meses, logrando tener un acompañamiento de la restauración hasta el momento del recambio de dentición (Figura 5 y 6).

Lo anterior permitió al paciente el restablecimiento de la función masticatoria, respiratoria y estética. La madre hace referencia a que se presenta una correcta trituración y deglución de alimentos, mejorando su digestión. La función respiratoria y estética se observa clínicamente mediante el sellado labial. Además, lo anterior mejoro la interacción con su familia y en la escuela.

\section{Discusión}

La Organización Mundial de la Salud informa que la prevalencia de caries en niños de edad preescolar es del $60 \%$ al $90 \%$. Para disminuir está prevalencia se 


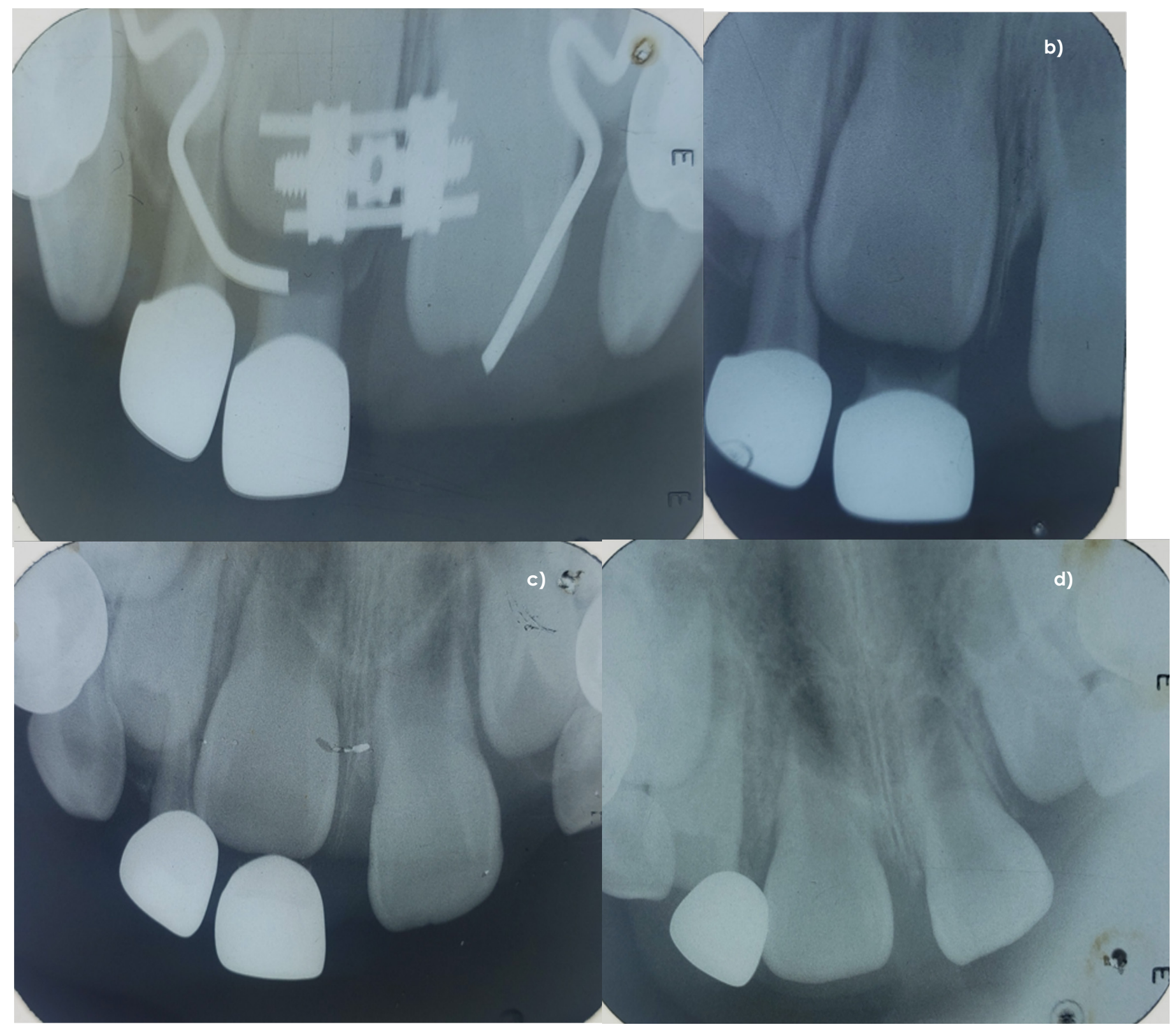

Figura 5. Imágenes radiográficas de seguimiento: a) seis meses, b) doce meses, c) dieciocho meses y d) veinticuatro meses de evolución.

requiere comprender la etiología y factores de riesgo para el desarrollo de la caries, así como capacitación y manejo de la biotecnología y de los biomateriales que se acoplen de la mejor forma a las necesidades de cada paciente. ${ }^{1,2}$

La presencia de enfermedades y trastornos bucales puede producir un impacto en la calidad de vida de los niños en edad preescolar; afectando su salud y bienestar emocional. ${ }^{2,3}$ Además, el tratamiento para cada paciente debe ser determinado por un buen diagnóstico que incluya el examen clínico y radiográfico, así como la cooperación de los padres hacia la adopción de hábitos que disminuyan los factores de riesgo, reeducando y evaluando la respuesta al tratamiento restaurativo de forma semestral. ${ }^{5}$

En este estudio se realizó un diagnóstico de forma adecuada, utilizando para el tratamiento coronas de zirconia. El material utilizado tienes ventajas de tener un colocar más natural y la durabilidad. Nuestros resultados coinciden con lo reportado en la literatura, en la que se 


\section{( @ RevistaCyRS}

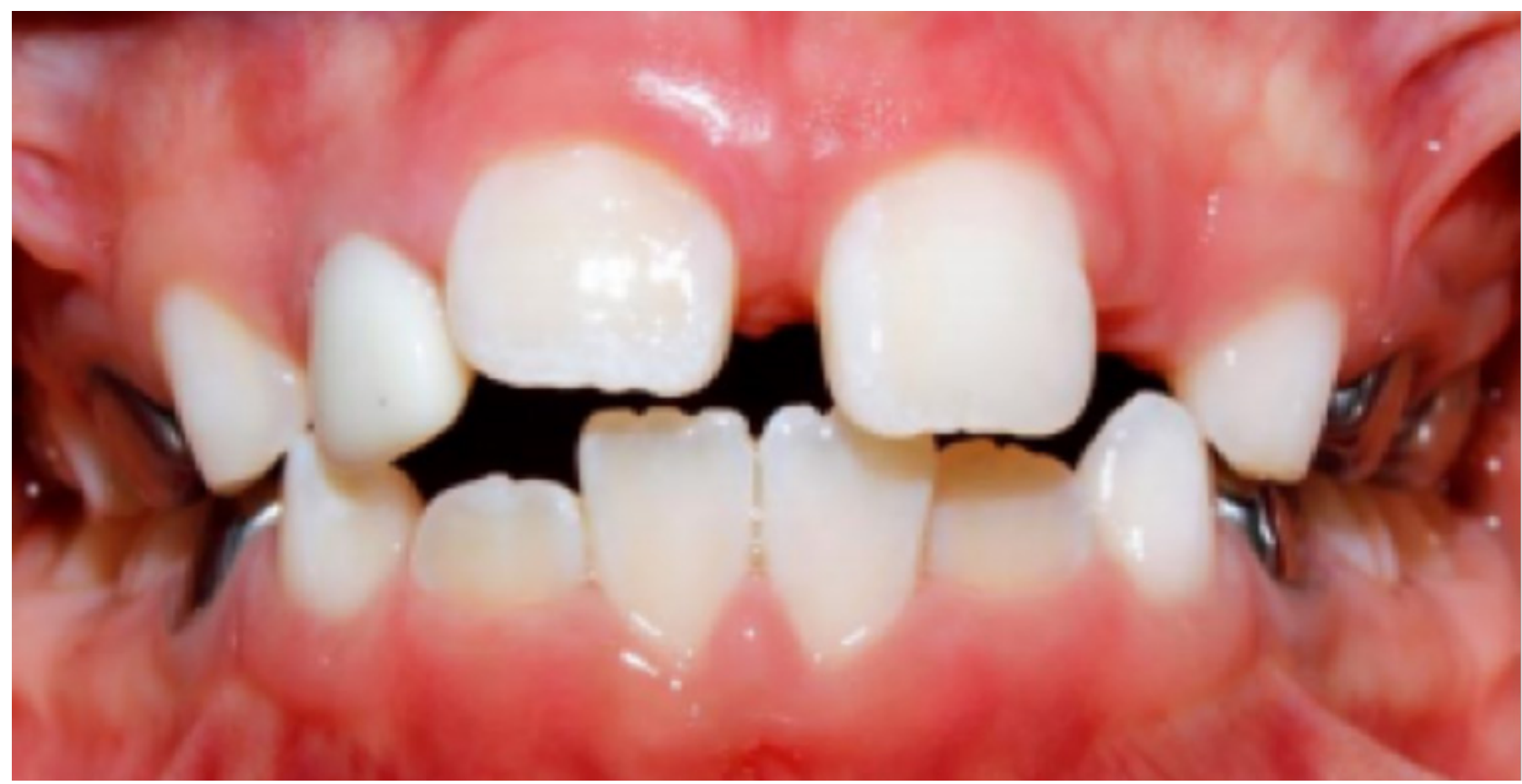

Figura 6. Fotografía vista frontal la cual nos muestra la erupción total de centrales superiores permanentes a los 24 meses de seguimiento clínico.

reporta que las coronas de zirconia son mayor resistencia y durabilidad, poco desgaste, traslucidez adecuada, biocompatible, esterilización por calor y larga duración. Sin embargo, no es recomendable en zonas de hemorragia o en pacientes con bruxismo. ${ }^{16}$

Una de las desventajas de las coronas de Zirconia es que no es posible retocarlas como las de acrílico. ${ }^{16} \mathrm{En}$ nuestro estudio se realizó un buen diagnóstico por lo que no sé presentó ninguna complicación al respecto. Para evitar complicaciones en el tratamiento con nuevos materiales es necesario utilizarlos con las técnicas adecuadas que cada fabricante recomienda; así como establecer planes de tratamiento personalizados de acuerdo con la necesidad de cada paciente; y vigilar los cambios que presentan al estar en función dentro de la cavidad oral. ${ }^{4,6}$

Las coronas de zirconia cuentan con una gran gama de colores, es de fácil fabricación y permite dejar un espacio libre de cerómero para lograr un buen ajuste coronal, por lo que ha sido reportadas sus ventajas en otros casos clínicos. ${ }^{17}$ El seguimiento a los 24 meses tampoco se presentaron reacciones adversas. Además, se logró mejorar la capacidad masticatoria, digestiva, estética y social en un infante, por lo que se observó un éxito clínico y funcional del tratamiento empleado.
Las coronas de Zirconia son un material que permite una estética adecuada y que tienen amplia durabilidad, por lo que es una buena alternativa para pacientes pediátricos. Sin embargo, es necesario realizar estudios en el que se compare con otros materiales, así como especificar las características de los pacientes, debido a que, en zonas de oclusión complicada o zonas de hemorragia se recomienda el uso de coronas de acrílico. ${ }^{16}$

\section{Conclusiones}

El uso de restauraciones estéticas de zirconia en dientes temporales anteriores en pacientes pediátricos, no presenta complicaciones y es una opción de tratamiento a un seguimiento de 24 meses, sin requerir terapia pulpar previa. Este tratamiento es aceptado de forma adecuada por los padres y el niño, ya que también mejoró su funcionalidad masticatoria y social. Sin embargo, es necesario realizar más estudios en los que se compare con otros materiales. 


\section{Referencias}

1. Edelstein BL. The dental caries pandemic and disparities problem. BMC Oral Health. 2006;6 Suppl 1(Suppl 1):S2. doi: 10.1186/1472-6831-6-S1-S2.

2. Abanto J, Carvalho TS, Mendes FM, Wanderley MT, Bönecker M, Raggio DP. Impact of oral diseases and disorders on oral health-related quality of life of preschool children. Community Dent Oral Epidemiol. 2011;39(2):105-114. doi: 10.1111/j.16000528.2010.00580.x.

3. Perazzo MF, Gomes MC, Neves ET, Martins CC, Paiva SM, Costa EMMB, Granville-Garcia AF. Oral problems and quality of life of preschool children: selfreports of children and perception of parents/caregivers. Eur J Oral Sci. 2017;125(4): 272-279.

4. Salami A, Walia T, Bashiri R. Comparison of parental satisfaction with three tooth-colored full-coronal restorations in primary maxillary incisors. J Clin Pediatr Dent. 2015; 39(5): 423-428.

5. Tinanoff N. and Douglass J.M. Clinical decision-making for caries management in primary teeth. $\mathrm{J}$ Dent Educ. 2001; 65(10): 1133-1142.

6. Peck S, Peck L. A time for change of tooth numbering systems. J Dent Educ.1993; 57(8): 643-647.

7. Garg V, Panda A, Shah J, Panchal P. Crowns in pediatric dentistry: a review. JAMDSR. 2016; 4(2): 41-46.

8. Walia T, Salami AA, Bashiri R, Hamoodi OM, Rashid F. A randomised controlled trial of three aesthetic fullcoronal restorations in primary maxillary teeth. Eur $\mathrm{J}$ Paediatr Dent. 2014;15(2):113-118.

9. Saha R, Malik P. Paediatric aesthetic dentistry: a review. Eur J Paediatr Dent. 2012;13(1):6-12.
10. Clark L, Martha H, Edward F. Comparison of amount of primary tooth reduction required for anterior and posterior zirconia and stainless-steel crowns. Pediatr Dent. 2016; 38(1): 42-46.

11. Ashima G, Sarabjot KB, Gauba K, Mittal HC. Zirconia crowns for rehabilitation of decayed primary incisors: an esthetic alternative. J Clin Pediatr Dent. 2014; 39(1): 18-22.

12. Woo D, Sheller B, Williams B, Mancl L, Grembowski D. Dentists' and parents' perceptions of health, esthetics, and treatment of maxillary primary incisors. Pediatr Dent. 2005; 27(1): 19-23.

13. Ramirez $\mathrm{H}$, Rangel E, Martínez $\mathrm{H}$, et al. Evaluación estética de seis tipos de coronas para dientes primarios. Revista de Odontopediatría Latinoamericana. 2017;7 (1): 6-15.

14. Villalobos $P$, Mendoza R, Yamamoto A, Alvear C. Uso de coronas de zirconio en el tratamiento de caries de la infancia temprana. Odontología Activa Revista Científica. 2018; 2(2): 23-30.

15. Medrano-González I, Esparza- Ramos F, RangelPadilla E, Yáñez-Acosta MF, Ramírez-Peña HA. Restauración estética del sector anterior en un paciente pediátrico. Rev Tamé. 2018; 7(19): 733-737.

16. Del Piñal L. Del Piñal I, Miegimolle M. Coronas estétics en odontopediatría. Odontol Pedíatr. 2019; 27(2): 137-149.

17. Pimentel EB, Trejo P, De León CS. Coronas de acero-cromo ceramizadas (Art-glass $\AA$ ) como una alternativa para la restauración de dientes temporales anteriores. Caso clínico. Rev Estomat. 2009; 17(1):26-29. 